\title{
Benefícios e impeditivos à integração da cadeia de suprimentos calçadista por meio da tecnologia de informação
}

\section{Benefits and impediments to the integration of the footwear supply chain using information tecnology}

\author{
Felipe Fehlberg Herrmann ${ }^{1}$ \\ Giancarlo Medeiros Pereira ${ }^{2}$ \\ Miriam Borchardt ${ }^{2}$ \\ Rosnaldo Inácio da Silva ${ }^{3}$
}

\begin{abstract}
Resumo: Este artigo analisa os benefícios e impeditivos à integração da cadeia de suprimentos calçadista por meio da tecnologia de informação. Um estudo de caso múltiplo, exploratório e qualitativo foi realizado na cadeia calçadista gaúcha. Foram investigados oito fornecedores de componentes (primeiro nível da cadeia, ex.: tacos, fivelas e adereços); oito empresas de manufatura de calçado (calçadistas ou montadoras do produto calçado); quatro organizações de varejo; e quatro prestadoras de serviço de TI. As técnicas de pesquisa utilizadas foram entrevista semiestruturada, observação direta e análise documental. A análise dos achados desvelou benefícios e impeditivos à integração da TI na referida cadeia de suprimentos. Identificou-se que dois modelos de integração da TI são adotados pelos prestadores de serviço de informática: proprietário (ou acessível apenas ao provedor de serviços) e aberto (aberto a todas as empresas). Apesar de fechado, o modelo proprietário de integração da TI é bastante disseminado, haja vista sua simplicidade e custo baixo. Em contrapartida, o modelo "aberto" propicia maior organização dos processos internos de gestão da TI, gestão de estoques e da produção. Os achados contribuem com a literatura ao desvelar elementos que podem criar condições para melhor articulação da cadeia de suprimentos calçadista, propiciando aumento de velocidade e flexibilidade produtiva ao setor. Apesar de atrativos, tais avanços podem ser dificultados por movimentos comerciais de empresas usuárias do modelo proprietário. A identificação desses impeditivos sugere a necessidade de novos estudos destinados a ampliar o entendimento acerca das disputas empresariais que se travam em meio a uma cadeia de suprimentos.
\end{abstract}

Palavras-chave: Integração da Tecnologia da Informação. Padronização dos processos. Parcerias. Cadeia de suprimentos calçadista.

\begin{abstract}
This article analyzes the benefits and impediments of the integration of the footwear supply chain through the adoption of information technology (IT). An exploratory qualitative multiple case study was carried out in a Footwear Supply Chain in Rio Grande do Sul (Gaúcha), Brazil. Eight accessory suppliers (first tier in the supply chain, such as: heels, buckles, and decorative ornaments), eight footwear manufacturing companies (footwear manufacturer or assembly companies), four retail companies, and four IT service providers. The research techniques used were semi-structured interviews, direct observation, and document analysis. The data analysis showed benefits and impediments to the integration of IT in the studied supply chain. It was found that two IT integration models have been adopted by the computer service providers: Proprietary (accessible only to the service providers) and Open (accessible to all companies) models. Although closed, the proprietary IT integration model is widely used given its simplicity and low cost. On the other hand, the Open Model enables higher organization in the internal processes of the IT management, inventory management, and production. The results obtained contribute to the literature since they show ways to improve Footwear Supply Chain development by increasing the speed and providing more productive flexibility to this sector. Although attractive, these developments can be hindered by commercial ventures of companies that adopt the Proprietary Model. The identification of these impediments suggests the need for further studies to increase the understanding of conflicts among supply chain partners.
\end{abstract}

Keywords: Information Technology Integration. Standardization process. Partnership. Footwear supply chain.

\footnotetext{
${ }^{1}$ Engenharia de Produção, Universidade Federal de Pelotas - UFPel, CEP 96010-610, Pelotas, RS, Brasil, e-mail: felipeherrmann@ outlook.com

2 Programa de Pós-graduação em Engenharia de Produção, Universidade do Vale do Rio dos Sinos - UNISINOS, CEP 93022-000, São Leopoldo, RS, Brasil, e-mails: gian@unisinos.br; miriamb@unisinos.br

${ }^{3}$ Programa de MBA em Gestão da Produção e Logística, Universidade do Vale do Rio dos Sinos - UNISINOS, CEP 95034-160, Caxias do Sul, RS, Brasil, rosnaldo@brturbo.com.br
}

Recebido em 5/11/2012 — Aceito em 1/10/2013

Suporte financeiro: Nenhum. 


\section{Introdução}

As empresas calçadistas brasileiras estão revendo seu posicionamento competitivo, de forma a explorar dimensões como: velocidade, qualidade do produto, flexibilidade produtiva, inovação, diversidade de modelos, ao invés de preço baixo e volume de produção (PEREIRA; SELLITTO; BORCHARDT, 2010). Conforme atesta a literatura, mudanças no posicionamento competitivo demandam o desenvolvimento de parcerias entre as empresas (SILVA; FISCHMANN, 1999; POWER, 2005). Entre os benefícios da parceria em diferentes setores, a literatura menciona: agilidade nas entregas (POWER, 2005; AVIV, 2007), redução dos níveis de inventário (YU; YAN; CHENG, 2001), redução de custos (YU; YAN; CHENG, 2001) e redução do efeito chicote (YU; YAN; CHENG, 2001). As parcerias envolvem: (i) a interligação de processos com vistas ao compartilhamento de informações; e (ii) a construção de infraestrutura de tecnologia da informação, permitindo a criação de novos conhecimentos (MALHOTRA; GOSAIN; El SAWY, 2005).

O reposicionamento competitivo também requer melhor exploração dos mecanismos dispostos pela tecnologia da informação, a qual, associada ao estabelecimento de parcerias, pode resultar nos seguintes benefícios para as partes: flexibilidade produtiva, incremento no compartilhamento de informações e melhoria no fluxo de materiais (CHEN; YANG; LI, 2007; SANDERS, 2008). Observa-se uma consolidação na literatura dos ganhos e benefícios advindos da integração da tecnologia da informação sobre o regime de parcerias (CACHON; FISHER, 2000; SAHIN; ROBINSON JUNIOR, 2005; NATIVI; LEE, 2012;). Hill e Scudder (2010) afirmam que significativos ganhos serão auferidos quando as organizações enfatizarem as parcerias com os fornecedores e clientes. Sahin e Robinson Junior (2005) mencionam que o avanço da tecnologia da informação está permitindo que as empresas reavaliem suas estratégias com a cadeia de suprimentos, explorando novas perspectivas de parceria interorganizacional. Da mesma forma, Nativi e Lee (2012) salientam a importância da tecnologia da informação no monitoramento em tempo real dos inventários e compartilhamento de informações na cadeia de suprimentos. Em outra linha de trabalho, Shamir (2012) destaca que o compartilhamento de dados entre os parceiros é essencial para a redução de estoques e custos. Zhou e Benton Junior (2007) apontam os diversos ganhos oriundos do compartilhamento de informações entre os parceiros. Trkman et al. (2007) e Chung, Tang e Ahmad (2011) mencionam que uma eficiente e eficaz integração da tecnologia da informação deve iniciar pela padronização dos processos. Thiesse et al. (2011) reportam ser crucial para a flexibilidade da infraestrutura de TI a padronização da identificação dos produtos e informações.

A necessidade de pesquisas afetas aos constructos integração da TI, padronização de processos e parceria é mencionada por diversos autores. Rai, Patnayakuni e Seth (2006) comentam a necessidade de se investigar a integração do fluxo físico, financeiro e de informação, e suas complementaridades, bem como o impacto destes a respeito do desempenho da empresa. Boeck e Wamba (2008) mencionam a importância de se identificar a qualidade da relação entre empresas que adotam o código de barras e EDI (electronic data identification). Azevedo e Carvalho (2012) evidenciam a relevância de esforços que promovam a difusão e a implantação em larga escala da TI no mercado da moda. Johansson e Palsson (2009) citam a relevância de se ampliar a compreensão acerca dos mecanismos de identificação, como forma de se melhorar o desempenho logístico. Barlas e Gunduz (2011) sugerem pesquisas para o desenvolvimento de estruturas de compartilhamento de informações em redes de abastecimento. Balocco et al. (2011) sugerem estudos que identifiquem os mecanismos por meio dos quais o aumento da visibilidade impacta a eficácia da cadeia de suprimentos. Münstermann, Eckhardt e Weitzel (2010) reforçam a importância de se investigar a existência de um efeito combinado entre a padronização dos processos e o compartilhamento de informações (por exemplo, troca eletrônica de dados, EDI). Seyal, Rahman e Mohammad (2007) sugerem a análise do compartilhamento de informações entre pequenas empresas.

A consideração simultânea das lacunas científicas indicadas no que se refere à uniformização de processos e à integração da TI sugere a seguinte questão de pesquisa: "Como se desenvolve a disputa pelo padrão dominante de gestão da informação na cadeia de suprimentos calçadista?" Espera-se, com isso, identificar e compreender os motivos da limitada padronização verificada, a despeito dos benefícios que esta pode trazer para a indústria calçadista. Para alcançar os objetivos do artigo, alguns pontos secundários necessitam ser atingidos, como segue: (i) identificar as informações e as abordagens em uso pelas empresas usuárias de cada um dos modelos de integração da TI; (ii) identificar os motivadores considerados pelas empresas usuárias para a adesão a cada um dos diferentes modelos de integração da tecnologia da informação atualmente em uso na cadeia de suprimentos; (iii) identificar restrições à adoção de cada um dos diferentes modelos de integração da tecnologia da informação atualmente em uso na indústria calçadista; e (iv) identificar as ações empreendidas pelas empresas usuárias e pelos provedores de soluções de TI aderentes a cada um dos modelos de integração da tecnologia da informação com vistas à perpetuação no mercado. 
Sob a ótica acadêmica, objetiva-se contribuir com estudos afetos à integração da TI (RAI; PATNAYAKUNI; SETH, 2006), especialmente, a troca eletrônica de dados e codificação dos produtos (BOECK; WAMBA, 2008) ligados ao mercado da moda (AZEVEDO; CARVALHO, 2012). Além disso, objetiva-se colaborar com entendimento acerca de como a padronização dos processos combinada com a TI pode apoiar a melhoria da eficiência das atividades e a eficácia dos processos (MÜNSTERMANN; ECKHARDT; WEITZEL, 2010), ao mesmo tempo que incrementam a visibilidade da cadeia de suprimentos (BALOCCO et al., 2011). A investigação também endereça a falta de trabalhos relacionados à tecnologia da informação na área da engenharia de produção (GODINHO FILHO; FERNANDES; LIMA, 2009).

Sobre a ótica empresarial, a elucidação da questão de pesquisa, ora proposta, propiciará que as lideranças da indústria calçadista identifiquem as variáveis que limitam a integração de processos entre as empresas com propósito de aumento de eficiência e competitividade. A identificação dos referidos elementos igualmente permitirá que se criem condições para melhor articulação da indústria calçadista, propiciando aumento da velocidade e flexibilidade produtiva ao setor, de forma a atender às dimensões competitivas, consideradas por Pereira, Sellitto e Borchardt (2010).

\section{Referencial teórico}

A investigação dos benefícios e impeditivos à integração da cadeia de suprimentos calçadista por meio da tecnologia de informação requer uma fundamentação teórica ampla. Assim, a primeira parte do referencial teórico conceitua cadeia de suprimentos, definidos os limites. Considerando-se que a gestão da informação em uma cadeia de suprimentos precisa ser orquestrada em regime de parceria entre clientes e fornecedores, este referencial apresenta um conjunto de trabalhos sobre o tema parcerias. A necessidade de padronizações nos formatos e processos suscitou a inserção do referencial acerca do tema padronização.
Lógica similar foi utilizada para a consideração do constructo troca eletrônica de informações. Os referidos constructos são apresentados a seguir. Ressalte-se que a troca eletrônica de informações de forma padronizada e aberta será doravante considerada como pertinente ao modelo aberto.

\subsection{Cadeia de suprimentos}

Fiala (2005) e Halldorsson et al. (2007) definem cadeia de suprimento como sendo o fluxo de bens e informações verificado desde a exploração da matéria-prima até o consumidor final. Esse fluxo de informações, materiais e de recursos financeiros se dá em ambas as direções entre as organizações envolvidas. Ballou (2000) apresenta uma estrutura que resume o escopo da cadeia de suprimentos, como visto na Figura 1. Corroborando com Ballou (2000), Tang e Musa (2011) enfatizam a integração das atividades da cadeia de suprimento num processo-chave ao invés do desenvolvimento de atividades individuais. Em adição, Chopra e Meindl (2007) e Hassini, Surti e Searcy (2012) definem cadeia de suprimento como sendo todas as etapas ou organizações envolvidas no atendimento das necessidades do cliente.

A gestão da cadeia de suprimentos, também conhecida por Supply chain Management (SCM), é responsável pelo gerenciamento das operações, customização e distribuição de bens, incluindo a administração da demanda, identificação de necessidades do cliente, transformação de matéria-prima e entrega dos bens gerados ao consumidor final (HALLDORSSON et al., 2007). Swaminathan e Tayur (2003, p. 1387) reforçam a definição de SCM, sendo: "[...] um gerenciamento eficiente dos processos end-to-end [...]", ou seja, uma gerência das atividades que compõem a cadeia de suprimentos, começando com o design do produto, ou serviço, e terminando com a sua venda, consumo ou descarte pelo consumidor. Complementam a definição, Hassini, Surti e Searcy (2012), como sendo o controle das operações da cadeia de suprimento, recursos e

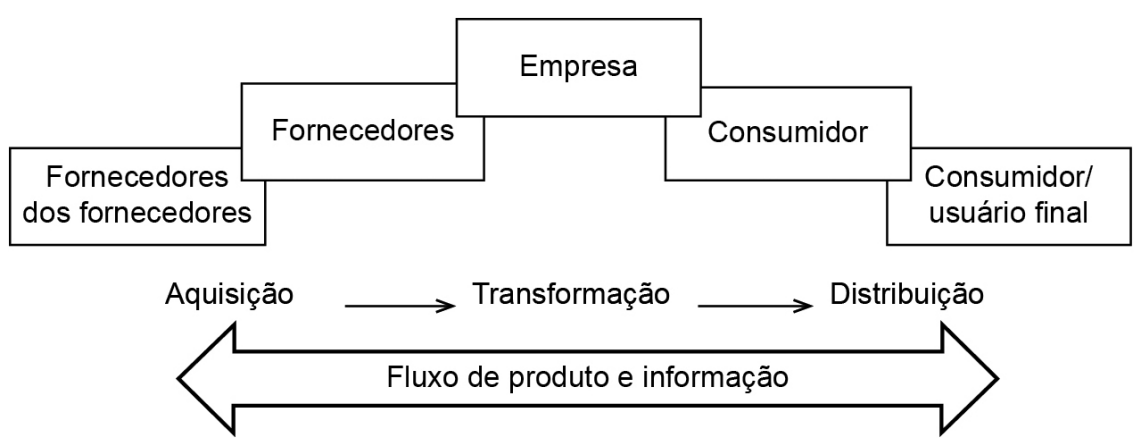

Figura 1. Estrutura da Cadeia de Suprimento. Fonte: Adaptado de Ballou (2000). 
informações, a fim de maximizar a rentabilidade da cadeia de suprimentos.

\subsection{Parcerias}

As parcerias se constituem em relacionamentos destinados a produzir benefícios individuais e coletivos (LI et al., 2005, p. 621). Parcerias em cadeia de suprimentos são fundamentais para o incremento da eficácia e da eficiência, para a adaptação às mudanças imprevistas, para o desenvolvimento de soluções e para a viabilização de economias de escala (SRINIVASAN; MUKHERJEE; GAUR, 2011). Parcerias estratégicas são formas de associação nas quais as empresas baseiam suas ações de longo prazo, compartilham benefícios/dificuldades mútuas, além de colaborar para a resolução de problemas (GUNASEKARAN; PATEL; TIRTIROGLU, 2001).

As parcerias podem mitigar eventuais deficiências dos demais elos de uma cadeia produtiva, reduzindo problemas como a falta de comunicação entre as partes. Além do mais, elas permitem a divisão de ganhos e a minimização de perdas (FIALA, 2005; AVIV, 2007). Por meio desta abordagem, a cadeia torna-se mais eficaz, pelas trocas de experiências, responsabilidades e tecnologias (YU; YAN; CHENG, 2001; AVIV, 2007). Ao mesmo tempo, os autores reforçam que a intenção da parceria é desenvolver operações e soluções "ganha-ganha" entre os membros de determinado setor. Apresenta-se ainda que, parcerias proporcionam uma diminuição no tempo de desenvolvimento de produtos e aumento da qualidade. No tocante aos benefícios advindos da parceria, alguns autores realçam os seguintes ganhos: agilidade no tempo de entrega/ recebimento dos produtos/insumos (POWER, 2005; RAI; PATNAYAKUNI; SETH, 2006); redução dos níveis de inventários (McLAREN; HEAD; YUAN, 2002; FIALA, 2005); redução de custos (AVIV, 2007); e redução do efeito chicote (FIALA, 2005).

\subsection{Padronização de processos}

A padronização dos processos é definida como sendo o grau em que trabalhos, políticas, operações, procedimentos são formalizados e seguidos (JANG; LEE, 1998; MÜNSTERMANN; ECKHARDT; WEITZEL, 2010). A qualidade do serviço ou do produto requer consistência nos processos da empresa. Essa consistência, por sua vez, é viabilizada pela padronização dos processos (UNGAN, 2006).

Com a padronização dos processos, a produção e os serviços tornam-se mais efetivos (UNGAN, 2006), logo, contribuem para o aumento da eficiência dos processos e atividades (MÜNSTERMANN; ECKHARDT; WEITZEL, 2010). Hsieh, Chou e Chen (2002) e Münstermann, Eckhardt e Weitzel (2010) reforçam que a padronização dos processos reflete positivamente na qualidade do serviço, visto que contribui para a redução da incerteza e a variabilidade nos processos (UNGAN, 2006). Além disso, o desenvolvimento de padrões contribui com aumento da performance operacional, porque elimina erros, reduz custos e facilita a comunicação (BEIMBORN et al., 2009). Ungan (2006) analisaram que a padronização de produtos, componentes e processos, contribui para a criação de novos produtos; desenvolvimento de abordagens para resolução de problemas; redução do número de componentes e atividades; além da redução de custos de armazenamento e transporte. Jayaram, Vickery e Droge (2000) reforçam que a padronização dos processos é uma variável importante para o desempenho da cadeia de suprimentos, mencionando ganhos na performance do Lead Time de fabricação, velocidade no tempo de entrega e capacidade de resposta ao consumidor.

\subsection{Compartilhamento de informações}

O compartilhamento de informações refere-se à troca de dados e informações proprietárias entre os parceiros (LI et al., 2005; SMITH; DUCHESSI; GARCIA, 2012). Como exemplos dessas informações compartilhadas, citam-se: previsões, programação de manufatura, nível de inventário, entre outros (SWAMINATHAN; TAYUR, 2003; SMITH; DUCHESSI; GARCIA, 2012). O compartilhamento de informações aumenta a visibilidade das atividades desenvolvidas entre as empresas, tornando mais eficientes as operações de suprimentos, fluxo de informações e materiais (SMITH; DUCHESSI; GARCIA, 2012). O impacto desse processo na cadeia de suprimentos é descrito por diversos autores. Jeong e Leon (2012) salientam a redução de distorções de informações; Nativi e Lee (2012) citam a possibilidade de maior integração entre as empresas; Smith, Duchessi e Garcia (2012) postulam, como ganhos, a redução de custos com inventário, aumento da visibilidade das atividades; Lee; So; Tang (2000) menciona a redução de custos com inventários e custos com manufatura.

A literatura explora a importância e os benefícios do compartilhamento da informação em diversos setores, como a redução de estoques, agilidade no atendimento, redução Bullwhip Effect, tempo de reposição (KÄRKKÄINEN; HOLMSTRÖM, 2002; JUN; CAI, 2003; AVIV, 2007), como também, realça a importância da qualidade (acuracidade, credibilidade, frequência) e do conteúdo (suficiência de dados e informação adequada) das informações compartilhadas (LI et al., 2005; ZHOU; BENTON JUNIOR, 2007; FAWCETT et al., 2007).

O compartilhamento de informações possibilita que estratégias de consumo ou produção sejam partilhadas com os diversos membros da cadeia de suprimentos (operadores downstream e upstream), como: Resposta 
rápida (Quick Response - QR); Resposta eficiente ao consumidor (Efficient Consumer Response - ECR); Gerenciamento do inventário pelo fornecedor (Vendor-Managed-Inventory - VMI); ou Programa de abastecimento contínuo (Continuous Replenishment Programs-CRP) (LEE; SO; TANG, 2000; SAHIN; ROBINSON JUNIOR, 2005; YUE; LIU, 2006; WU; CHENG, 2008). Salienta-se que o advento da internet e o aperfeiçoamento de softwares de troca eletrônica de dados na cadeia de suprimentos colaboraram para a melhoria do desempenho das operações e negócios, reduzindo custos, retrabalho e coordenação das atividades entre as empresas (SWAMINATHAN; TAYUR, 2003; SMITH; DUCHESSI; GARCIA, 2012). O compartilhamento de informações, por meio da TI, é considerado a melhor estratégia para conter o então chamado "Bullwhip Effect" (LEE; SO; TANG, 2000; FIALA, 2005).

\subsection{Troca eletrônica de informações}

A troca eletrônica de informações via EDI (Eletronic Data Interchange) constitui-se num meio eletrônico em que as informações críticas e específicas são comunicadas para a cadeia (LI et al., 2005). Seyal, Rahman e Mohammad (2007) mencionam algumas dessas informações: ordens de compra; guia de remessa; documentos aduaneiros; documentos do consumidor; informações de consumo; quantidade; e volume.

Importa salientar que EDI, em diversos setores, facilita o compartilhamento de estratégias de consumo e/ou vendas de produtos, resultando na redução do efeito chicote (Bullwhip Effect), e tornando a cadeia mais enxuta (Lean) (YU; WANG; LIANG, 2007). Wu e Cheng (2008) reforçam que o EDI surgiu do avanço da TI, aumentando significativamente a possibilidade de obtenção em tempo real de informações.

A codificação dos produtos por meio do código de barra representa uma forma de utilizar-se da TI para melhorar o fluxo de materiais e compartilhamento de informações dentro da cadeia de suprimentos (BOECK; WAMBA, 2008). Entretanto, para potencializar os ganhos advindos da codificação dos produtos, necessita-se adotar um padrão para essa codificação. Assim evitar-se-á a incompatibilidade na leitura e compartilhamento da informação (MURPHY, DARLEY, 1999; JUN; CAI, 2003; FAWCETT et al., 2007). Um dos padrões globais existentes para tal é o do código de barra, EAN (European Standards) (POWER, 2005). Este padrão é administrado pela empresa GS1, que está presente em mais de cem países. O código EAN é conhecido como "The global language of business", sendo também chamado de código GS1 (Global System).

O modelo de identificação GS1 permite que as informações sejam lidas e interpretadas por todas as organizações que adotam o modelo, eliminando a incompatibilidade na leitura dos códigos. O código de barras GS1 é formado por linhas pretas e brancas intercaladas, conforme Figura 2, que podem ser lidas por leitores de ópticos (BOECK; WAMBA, 2008). O código de barra é utilizado para aumentar a acurácia das informações, velocidade de transmissão dos dados, compartilhamento de informações e alimentação de sistemas de EDI. Diante disso, supre estratégias do tipo VMI (BOECK; WAMBA, 2008; PALSSON; JOHANSSON, 2009). Segundo Yu, Wang e Liang (2012) o VMI (Vendor Managed Inventory) é um sistema no qual o fornecedor administra os estoques de seus clientes/consumidores. O gerenciamento dos estoques é centralizado para reduzir o custo total de inventário na cadeia de suprimentos (YU; WANG; LIANG, 2012).

\section{Delineamento da pesquisa}

O método de pesquisa é o estudo de caso, no qual é investigado "Como se desenvolve a disputa pelo padrão dominante de gestão da informação na cadeia de suprimentos calçadista?" Em síntese, o presente estudo caracteriza-se por ter uma abordagem qualitativa, em que o método escolhido é o estudo de caso múltiplo, com propósito exploratório. A operacionalização na coleta de dados deu-se basicamente de três formas: entrevistas (semiestruturadas, com questões abertas) com os gerentes de TI e/ou suprimentos; documentação (arquivos, notas, ofícios e relatórios); e observações diretas (análise do processo de recebimento, expedição de mercadorias e execução da troca eletrônica de informações).

As unidades de análise são empresas calçadistas, seus fornecedores de insumos, varejistas e empresas prestadoras de serviços de TI. A seleção das empresas calçadistas deu-se por indicação da ABICALÇADOS (Associação Brasileira das Indústrias de Calçado). A seleção das calçadistas considerou as principais fábricas atuantes no Estado do Rio Grande do Sul. Os varejistas foram indicados pelos calçadistas. Os

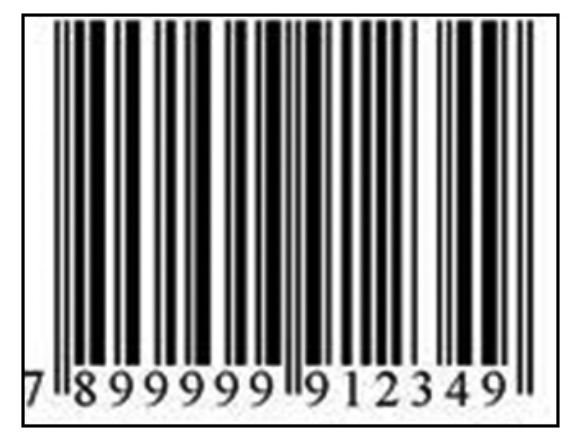

Figura 2. Código de barra - modelo GS1. Fonte: GOL (2012). 
fornecedores investigados são todos do primeiro escalão de relacionamento, ou seja, fornecedores diretos de componentes para as referidas indústrias calçadistas. Eles também foram indicados pelos calçadistas. As prestadoras de serviço de TI foram selecionadas com base no relacionamento comercial com as empresas calçadistas, varejistas e fornecedores de insumos (componentes). As empresas de TI selecionadas detêm aproximadamente $90 \%$ do mercado de serviços de TI na indústria calçadista do Rio Grande do Sul. Assim, o tamanho da amostra foi de vinte e quatro empresas.

Para a análise dos resultados, usaram-se os conceitos emanados por Fleury et al. (2012) e Bardin (2002). Num primeiro estágio, foi realizada a redução dos dados, utilizando-se somente as informações que foram fundamentais para a pesquisa, possuindo ligação direta com o tema e constructos. Esses dados foram oriundos das transcrições das narrativas de entrevistas, documentos coletados e observações. Como o estudo caracteriza-se quanto ao seu propósito exploratório e quanto ao "caso" múltiplo, após a etapa de organização dos dados, utilizou-se a confrontação dos "casos" em estudo. A organização dos dados foi por meio da codificação, na qual códigos representaram as narrativas e documentos ligados aos constructos e à teoria do presente trabalho. Depois dessa etapa, foi possível identificar as convergências e divergências entre as fontes e evidências. Consequentemente, viabilizou-se o entendimento da questão de pesquisa. O modelo analítico utilizado para análise dos dados foi a análise de conteúdo, descrevendo de forma objetiva, sistemática e qualitativa o conteúdo colhido nos trabalhos de campo (BARDIN, 2002).

Previamente, na apresentação das etapas envolvidas na construção do trabalho, esboçam-se as fases que originaram a presente investigação. Por meio de contatos com lideranças da indústria calçadista, observou-se uma falta de uniformidade na integração da tecnologia da informação entre os membros da cadeia de suprimentos calçadista. Numa segunda etapa, realizaram-se visitas de campo com perguntas abertas para analisar in loco os problemas da limitação da integração da TI na cadeia de suprimentos calçadista. Nessa etapa, realizaram-se visitas a duas empresas de manufatura do setor. As empresas foram escolhidas conforme a facilidade de acesso e por ambas serem referência na adoção de melhores práticas logísticas na indústria calçadista. Em um estágio posterior, buscou-se fazer uma leitura horizontal da teoria que abordava o tema. Por último, reuniram-se todos os dados e achados de campo para discussão com especialistas e pesquisadores que estão ligados diretamente ao tema de pesquisa. Logo, conseguiu-se estruturar o problema de pesquisa e "casos" alvos desta. O restante da pesquisa é descrito na Figura 3.

Finalizando, o presente trabalho caracteriza-se por ter uma abordagem qualitativa, na qual o método escolhido foi o estudo de caso múltiplo, com propósito exploratório. A operacionalização deu-se por meio de entrevistas (semiestruturadas), análise documental e visita de campo. Os achados advieram de vinte e

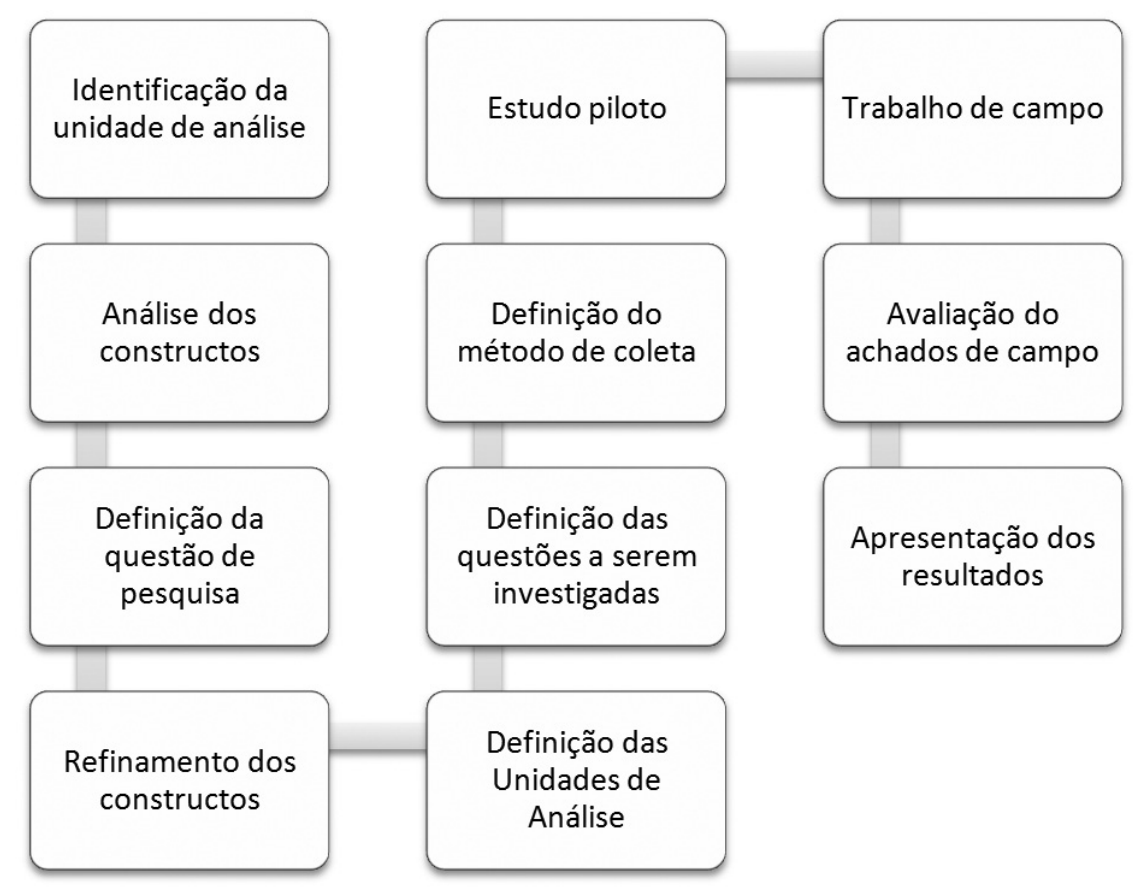

Figura 3. Fases do método de trabalho. Fonte: Própria dos autores. 
quatro empresas da cadeia calçadista da região do Vale do Rio dos Sinos, Rio Grande do Sul - Brasil.

\section{Análise e discussão dos dados}

\subsection{Modelo proprietário x modelo aberto}

A codificação dos produtos na cadeia de suprimentos calçadista é contemplada por dois modelos distintos, a saber: o modelo proprietário e o modelo aberto. $\mathrm{O}$ Quadro 1 sintetiza os achados com relação aos dois modelos identificados, ressaltando as divergências e convergências.

Nas próximas seções, uma análise e discussão acerca das diferenças entre os modelos de integração da TI, listados neste estudo, serão exploradas de forma específica, explorando pontos como compartilhamento de informações, padronização dos processos, avaliação dos modelos e determinantes pela opção empresarial.

\subsection{Compartilhamento de informações}

Análises indicam que o compartilhamento de informações nos modelos investigados pode ser agrupado nas seguintes dimensões: quantidade de informações transitadas, estratégias de operações logísticas desenvolvidas (gerenciamento de estoque e automação dos processos de compra) e integração dos sistemas de TI. O Quadro 2 resume os achados com relação aos dois modelos citados.

As diferenças entre os modelos de compartilhamento de informações investigados podem ser divididas em três grupos, a saber: quantidade de informações transitadas, estratégias das operações logísticas e integração das empresas por meio dos sistemas de TI.

Em se tratando de quantidade de informações, o modelo proprietário permite a partilha de apenas quatro informações, como: lista de preços, pedido de compra, aviso de despacho e nota fiscal. Essa limitação advém da concepção original do modelo, a saber: o foco no gerenciamento da produção e dos estoques. Em adição a isso, salienta-se o modelo proprietário, no qual não se seguem normas ou padrões específicos. Isso dificulta a integração da TI no setor. Fawcett et al. (2007) e Thiesse et al. (2011) identificaram que a falta de padrões é uma das barreiras para o avanço da integração da TI. Wang, Tai e Wei (2006) identificaram a necessidade de substituição de modelos proprietários de integração da TI por um padrão aceito globalmente. $\mathrm{O}$ modelo aberto é um tipo de modelo aceito globalmente, o qual apoia o controle interno, além de permitir a identificação do produto de forma mais abrangente. Nele, maior número de informações é compartilhado: alteração do pedido, notificação de recebimento, características do produto e aviso de pagamento. Smith, Duchessi e Garcia (2012) postulam que o compartilhamento de informações por um recurso uniforme torna as operações de suprimento mais eficientes, bem como facilita o fluxo de informações e materiais entre os diferentes agentes de uma cadeia de suprimentos.

No tocante ao desenvolvimento conjunto de estratégias, o presente estudo não identificou a estruturação de estratégias de operações logísticas entre os usuários do modelo proprietário. No modelo aberto, observou-se o desenvolvimento de estratégias de operações logísticas, como: gerenciamento de estoques (VMI) e automação dos processos de compra. O desenvolvimento de estratégias de compra e estoque no cenário (característico da indústria calçadista estudada), Make-to-order, é citado por diversos autores como fundamental para o atendimento ao cliente (SAHIN; ROBINSON JUNIOR, 2005; YUE; LIU, 2006). Essas estratégias podem alavancar o desempenho da gestão de compras, provendo substancial melhora da performance financeira (SAHIN; ROBINSON JUNIOR, 2005).

A análise dos achados afetos à integração da TI no modelo proprietário revelou que esta precisa ser viabilizada por uma terceira organização (a VAN). Essa empresa realiza a interpretação e a retransmissão das informações conforme as especificações de cada modelo proprietário. Essa intermediação agrega custos para as empresas usuárias do modelo proprietário. Tal contexto não foi observado junto às empresas do modelo aberto. Nessas, a integração da TI é

Quadro 1. Sumário dos achados - Modelo proprietário x aberto.

\begin{tabular}{|c|c|}
\hline MODELO PROPRIETÁRIO & MODELO ABERTO \\
\hline $\begin{array}{l}\text { Atende às necessidades operacionais básicas (produção e } \\
\text { estoque) da empresa; } \\
\text { Limitado compartilhamento de informações entre os } \\
\text { parceiros de negócio; } \\
\text { Não atende às normas ou padrões específicos de } \\
\text { compartilhamento de informações; } \\
\text { Compartilhamento viabilizado pela intermediação da } \\
\text { VAN (Value Addition Network). }\end{array}$ & $\begin{array}{l}\text { Atende às necessidades operacionais básicas (produção e } \\
\text { estoque) da empresa; } \\
\text { Permite o controle operacional da empresa; } \\
\text { Baseado em um padrão aberto (modelo GS1); } \\
\text { Amplo compartilhamento de informações entre os } \\
\text { usuários do modelo; } \\
\text { Permite a adoção da automação alinhada aos processos; } \\
\text { Não necessita da interferência da VAN para o } \\
\text { compartilhamento de informações. }\end{array}$ \\
\hline
\end{tabular}

Fonte: Própria dos autores. 
viabilizada pela facilidade na troca de dados entre as empresas usuárias do referido modelo. Essas trocas de informações são feitas diretamente entre as empresas interessadas e baseiam-se na ISO 9735. Isso dispensa a interferência de uma terceira organização como a $V A N$ na troca eletrônica de dados $(E D I)$. A análise desses achados indica que o modelo aberto mostra-se mais atrativo em termos de flexibilidade de integração da TI (SANDERS, 2005; YUE; LIU, 2006). Além do mais, também contribui para o incremento da parceria entre as empresas (YU; YAN; CHENG, 2001), nos moldes do proposto por Power (2005).

\subsection{Padronização de processos}

Identificou-se que os processos de compra (expedição e recebimento de produtos) de cada um dos modelos investigados possuem divergências na sequência de atividades operacionais e administrativas. O Quadro 3 resume os achados relativos à padronização dos processos entre os modelos de integração da TI estudados.

Nas visitas e na análise dos documentos de processos, também foi possível identificar que o modelo aberto faz uso de uma sequência padronizada de atividades operacionais e administrativas que abarcam desde a identificação do produto até o recebimento da mercadoria. $\mathrm{O}$ resultado desta etapa permitiu a elaboração do seguinte fluxo de processo (Figura 4):
Os achados do presente estudo não desvelaram nenhum padrão entre os processos de compra atualmente em uso pelas empresas usuárias do modelo proprietário. A falta de padronização parece ser a gênese das importantes inconsistências identificadas entre o estoque real, ou os dados da nota fiscal, e o montante descrito no sistema de informações alinhadas com o modelo proprietário. Tal cenário não se verificou no modelo aberto, no qual se identificou uma padronização dos processos de compra (expedição e recebimento). Constatou-se que a padronização propiciada pelo modelo aberto evita a sobreposição de atividades, a realização de retrabalhos e as falhas operacionais e administrativas. Análises documentais revelaram a existência de uma descrição textual das atividades do processo de compra junto às empresas usuárias do modelo aberto. Essa documentação está alinhada com os postulados de Trkman et al. (2007), os quais destacam a importância da existência de diagramas associados à descrição textual, como requisito para uma eficiente e eficaz integração dos processos. A necessidade de padronização dos processos de compra é destacada por Chung, Tang e Ahmad (2011).

\subsection{Avaliação do modelo proprietário}

A falta de um padrão não apenas para a codificação de produtos, mas também para a estruturação de processos verificada no modelo proprietário demanda a reetiquetação do produto a cada novo elo da cadeia

Quadro 2. Diferenças no compartilhamento de informações.

\begin{tabular}{|l|l|l|}
\hline \multicolumn{1}{|c|}{ ELEMENTO } & \multicolumn{1}{|c|}{ MODELO PROPRIETÁRIO } & \multicolumn{1}{c|}{ MODELO ABERTO } \\
\hline $\begin{array}{l}\text { Quantidade de } \\
\text { Informações }\end{array}$ & $\begin{array}{l}\text { Lista de preços; pedido de compra; aviso } \\
\text { de despacho; nota fiscal. }\end{array}$ & $\begin{array}{l}\text { Todas as informações do modelo } \\
\text { proprietário mais: alteração de pedido; } \\
\text { aviso de recebimento; características de } \\
\text { produto; aviso de pagamento. }\end{array}$ \\
\hline \multirow{2}{*}{ Estratégias } & $\begin{array}{l}\text { Não observado o compartilhamento de } \\
\text { estratégias de operações logísticas. }\end{array}$ & $\begin{array}{l}\text { Identificados projetos conjuntos para a } \\
\text { automação dos processos de compra e } \\
\text { gerenciamento de estoques. }\end{array}$ \\
\hline de TI & $\begin{array}{l}\text { Integração dos sistemas de TI por } \\
\text { intermédio da VAN. }\end{array}$ & $\begin{array}{l}\text { Troca de informações pertinentes aos } \\
\text { negócios entre sistemas de gestão } \\
\text { diferentes, sem a interferência de VAN'S. }\end{array}$ \\
\cline { 2 - 3 } & $\begin{array}{l}\text { Sem padronização no trânsito eletrônico } \\
\text { de dados (EDI). }\end{array}$ & $\begin{array}{l}\text { Mensagens (EDI) padronizadas pela ISO } \\
\text { 9735. }\end{array}$ \\
\hline
\end{tabular}

Fonte: Própria dos autores.

Quadro 3. Diferenças nos processos de compra.

\begin{tabular}{|l|l|}
\hline \multicolumn{1}{|c|}{ MODELO PROPRIETÁRIO } & \multicolumn{1}{c|}{ MODELO ABERTO } \\
\hline Falta de padronização no processo de compra; & Processo de compra padronizado entre as empresas \\
Inconsistência entre o estoque físico e os dados acerca & usuárias do sistema; \\
do mesmo estoque, descrito no sistema de informação & Restrita existência de erros entre o estoque físico e o \\
(até 8\%); & descrito no sistema de informações da empresa; \\
Presente em 90\% dos varejistas. & Presente em 10\% dos varejistas. \\
\hline
\end{tabular}

Fonte: Própria dos autores. 
produtiva ou comercial. A necessidade de reetiquetação pode resultar em erros, o que, conforme identificado nas entrevistas, acaba por gerar novas inconsistências entre o estoque físico e o declarado no sistema de informações. Entrementes, a despeito dos problemas descritos, as empresas que fazem uso do modelo proprietário entendem que ele é atrativo para suas organizações. O Quadro 4 sintetiza os ganhos e problemas encontrados pelas empresas usuárias do modelo proprietário.

As análises das entrevistas indicam que as grandes empresas optaram pelo modelo proprietário em função dos seguintes elementos: simplicidade e quantidade reduzida de informações transitada entre as empresas usuárias do modelo; ampla base instalada; abordagem comercial dos provedores de solução de TI; menor custo na aquisição dos códigos de barra e manutenção/ instalação de softwares de compartilhamento de informações. Esses elementos mitigaram a influência de outros pontos negativos existentes no modelo proprietário como: a necessidade de manutenção de múltiplos sistemas de TI, o custo de reetiquetação e a não adesão a algum padrão global. Com efeito, a ponderação dos elementos listados parece ter sido favorável ao modelo proprietário em $98 \%$ das empresas do setor, especialmente entre as pequenas empresas. A existência de múltiplas empresas líderes na indústria calçadista acabou dando origem a uma série de modelos proprietários, cada um associado a uma grande empresa do setor. Esses achados divergem da proposta de Shamir (2012), a qual sugere que as empresas devem unificar seus modelos de compartilhamento de informações com o objetivo de aumentar sua eficiência operacional e reduzir seus custos com o compartilhamento de informações.

A opção pelo modelo proprietário por parte da maioria das pequenas e médias empresas também pode ser creditada à influência das empresas líderes, ou dos maiores clientes do setor. Conforme identificado, vários são os casos de fornecedoras que convivem com mais de um modelo proprietário, objetivando atender às demandas de seus clientes. A busca pelo alinhamento com as empresas líderes segue uma tendência já apresentada na literatura (SANDERS, 2005). A busca pelo alinhamento por parte das organizações fornecedoras também pode ser creditada à falta de recursos e necessidade de extensivo conhecimento para a adoção de outros modelos, nos moldes do descrito por Thiesse et al. (2011).

A opção dos provedores de TI pelo modelo proprietário pode ser creditada à combinação entre: o atendimento da demanda das empresas líderes; a

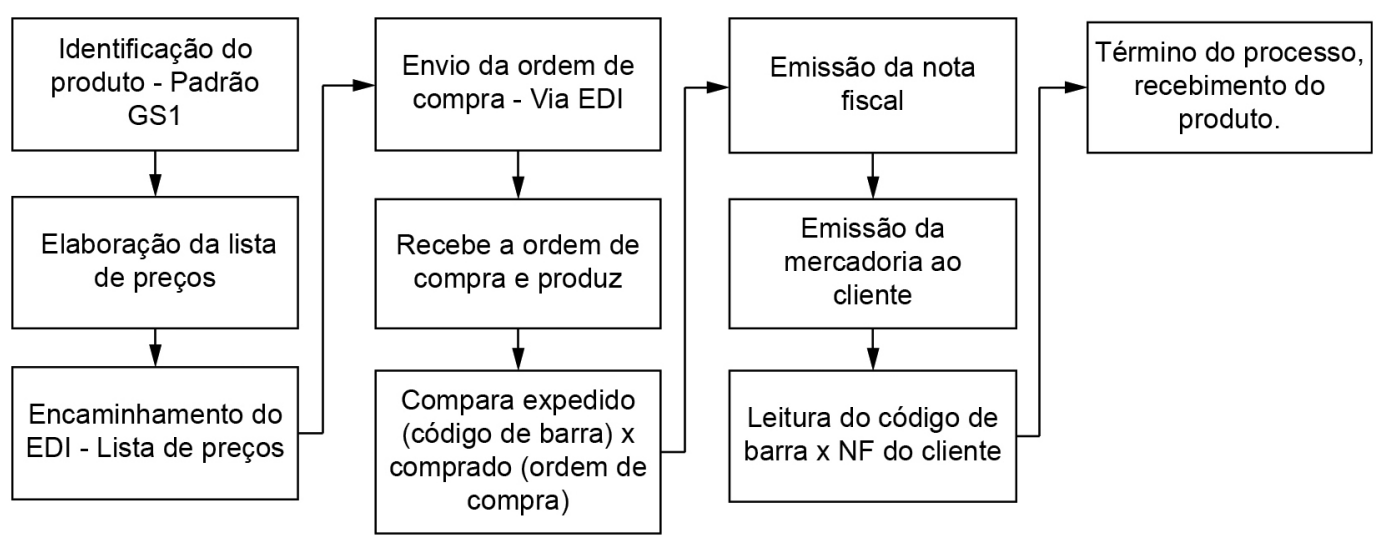

Figura 4. Processo de compra no modelo aberto. Fonte: Própria dos autores.

Quadro 4. Sumário dos achados - Avaliação do modelo proprietário.

\begin{tabular}{|c|l|}
\hline ELEMENTO & \multicolumn{1}{c|}{ MODELO PROPRIETÁRIO } \\
\hline \multirow{3}{*}{ Ganhos } & $\begin{array}{l}\text { Simplicidade e pequena quantidade de informações transitadas entre as empresas; } \\
\text { Menor custo de softwares e licenças de códigos de barra; } \\
\text { Alinhamento com os sistemas em uso pelos clientes. }\end{array}$ \\
\hline \multirow{3}{*}{ Problemas } & $\begin{array}{l}\text { Reetiquetação de produtos onera o custo do produto entre 1,2\% e 1,5\% do preço de venda na } \\
\text { indústria; } \\
\text { Limitada integração da TI com empresas fornecedoras e com clientes dentro da cadeia de } \\
\text { suprimentos; } \\
\text { Fornecedores precisam manter diversos modelos proprietários; } \\
\text { Inconsistências nos dados de estoque podem chegar a 8\%; } \\
\text { Atrasos no envio das etiquetas resultam em custos adicionais para os fornecedores. }\end{array}$ \\
\hline
\end{tabular}

Fonte: Própria dos autores. 
simplicidade do modelo proprietário; a inexistência de restrições ou custos para a obtenção de códigos de identificação; a busca da fidelização dos clientes; o limitado acesso que as pequenas empresas prestadoras de serviços de TI têm às informações necessárias à elaboração e manutenção dos softwares. Além disto, os provedores de TI alinhados com o modelo proprietário manifestaram certo receio quanto aos reais benefícios advindos da eventual adoção do modelo aberto de gestão das informações. Segundo estes, poucos são os casos de sucesso do modelo aberto no setor calçadista em termos de aplicabilidade e funcionalidade. A relação entre a adesão a um modelo de TI e sua adoção pelas empresas foi investigada por Fawcett et al. (2007). Por último, identificou-se que as prestadoras de serviços de TI aderentes ao modelo proprietário temem perder receitas advindas das customizações e adaptações caso adotem o modelo aberto. Isso porque o modelo aberto dispensa tais adaptações. Os provedores de TI do modelo proprietário também receiam que a adoção de padrões globais venha a eliminar as vantagens competitivas que têm ante seus concorrentes. Conforme verificado, a remoção dessas vantagens implicaria a redução das margens de ganho dos provedores de TI alinhada com dado modelo proprietário.

\subsection{Avaliação do modelo aberto}

Análises documentais revelam que aproximadamente $2 \%$ das empresas da cadeia de suprimentos calçadista adotam o modelo aberto. Apesar do baixo número de usuários, os entrevistados de ambos os modelos (proprietário e aberto) entendem que o modelo aberto se constitui na melhor opção técnica para as empresas da cadeia de suprimentos calçadista. O Quadro 5 resume os ganhos e problemas listados pelos entrevistados.
A análise dos elementos que referendaram a opção pelo modelo aberto por parte das grandes empresas da indústria calçadista permite que se identifiquem dois grandes grupos, a saber: o aprimoramento das informações e processos; o pleno aproveitamento das possibilidades de automação providas pelo novo sistema, especialmente no tocante ao desenvolvimento de estratégias de operações logísticas e projetos conjuntos.

Em se tratando do aprimoramento das informações e processos, o presente estudo identificou que as empresas optantes pelo modelo aberto objetivaram aprimorar a padronização de processos; incrementar o compartilhamento de informações com outras empresas da cadeia; simplificar o controle dos estoques. A padronização possibilitou melhor codificação do produto e simplificação do processo de compra. Os ganhos listados pelos entrevistados das empresas usuárias do modelo aberto estão alinhados com os ganhos descritos na literatura. Em linhas gerais, esses ganhos são: diminuição de atividades operacionais e administrativas, redução do nível de inventário e aumento da acuracidade de informações (JUN; CAI, 2003); aumento da visibilidade das atividades ligadas ao fluxo de serviços e produtos (SMITH; DUCHESSI; GARCIA, 2012). Corroborando, Jeong e Leon (2012) salientam a redução de distorções de informações compartilhadas entre os parceiros; Nativi e Lee (2012) citam a possibilidade de maior integração entre as empresas; Lee; So; Tang (2000) menciona o maior controle dos estoques e redução de custos com inventários e custos com manufatura.

A automação se constitui no segundo grupo de elementos considerado pelas grandes empresas, quando da opção pelo modelo aberto. Conforme verificado, as empresas almejam agilizar o tempo de reposição de insumos e de produtos acabados. Tal preocupação com agilidade no tempo de reposição converge com

Quadro 5. Sumário dos achados - Modelo aberto.

\begin{tabular}{|c|c|}
\hline ELEMENTO & MODELO ABERTO \\
\hline Problemas & $\begin{array}{l}\text { Limitado número de empresas utilizando o modelo aberto ( } 2 \% \text { do setor); } \\
\text { Dificuldades administrativas junto a GS1 para aquisição dos códigos de barra; } \\
\text { Custos com a aquisição e manutenção dos códigos de barra, especialmente, para as pequenas } \\
\text { empresas; } \\
\text { Custo elevado com a adequação do sistema de TI ao modelo aberto; } \\
\text { Demora na aquisição de códigos de barra por parte da GS1; } \\
\text { Falta de dados técnicos para o desenvolvimento de softwares; } \\
\text { Desconhecimento de fontes de pesquisa de dados para elaboração de software adequado ao } \\
\text { padrão internacional. }\end{array}$ \\
\hline Ganhos & $\begin{array}{l}\text { Facilidade na integração dos sistemas de TI; } \\
\text { Apoio na padronização dos processos de compra, codificação dos produtos e troca eletrônica } \\
\text { de dados; } \\
\text { Redução drástica dos erros de expedição; } \\
\text { Redução de estoque e do quadro de funcionários. }\end{array}$ \\
\hline
\end{tabular}

Fonte: Própria dos autores. 
o trabalho de Hilsdorf, Rotondaro e Pires (2009), que citam a afirmativa que, no contexto da nova economia, a agilidade e a capacidade de oferecer prazos de entrega cada vez mais curtos tornaram se condições valorizadas pelo cliente. Para tanto, foi de crucial importância a possibilidade de padronização existente no modelo aberto (padronização de processos e sistemas de TI, entre as empresas parceiras). A associação entre a padronização e a automação melhorou a eficiência operacional e administrativa das empresas usuárias do modelo aberto, especialmente no tocante à automação dos processos de compra. A possibilidade de associação do GTIN do calçado com os diversos GTINs de componentes proporcionada pelo modelo aberto viabilizou a automação dos processos de compra de componentes.

Os benefícios proporcionados pela automação vão ao encontro do trabalho de Thiesse et al. (2011), especialmente no tocante à eliminação de trabalhos manuais e conferências. Outros elementos pertinentes à implantação de soluções automatizadas também foram encontrados, como: a disponibilização de um ambiente on line (web based) para o compartilhamento de informações (SMITH; DUCHESSI; GARCIA, 2012); e a divulgação de casos de sucesso entre as parceiras (FAWCETT et al., 2007). Cumpre destacar que todas as ações relacionadas à automação foram orquestradas conjuntamente entre os parceiros de negócio da cadeia de suprimentos calçadista, por exemplo, o VMI (BOECK; WAMBA, 2008; PALSSON; JOHANSSON, 2009).

\subsection{Determinantes da opção empresarial}

As empresas usuárias dos dois modelos investigados apresentaram diferentes motivos para justificar sua opção por dado modelo. As optantes pelo proprietário, especialmente as fornecedoras, o fizeram por exigências dos clientes. As usuárias do modelo aberto o fizeram objetivando reduzir o número de atividades e pessoas, agilizar processos (compra, recebimento e expedição), melhorar o nível de acuracidade das informações e gestão de estoques. A possibilidade de redução dos custos de reetiquetação dos produtos e a melhoria das informações transitadas (EDI) também foram citadas pelas empresas que adotam o modelo aberto de integração da TI.
Os provedores de soluções alinhadas com o modelo proprietário declararam ter optado por ele em função da facilidade de obtenção, manutenção e elaboração do código de identificação do produto e trânsito de mensagens eletrônicas. Essas empresas salientam a importância do custo de aquisição da licença de utilização do código de barra junto à GS1 com um indutor aos modelos proprietários.

Os provedores de soluções alinhadas com o modelo aberto consideram-no mais adequado em função das possibilidades de eliminação da VAN no trânsito de informações, reduzindo, assim, custos com a manutenção de TI e eliminando erros nas informações transitadas. Exemplos de erro em informações são: erro na importação das mensagens, data inválida, regra de negócio infligida, CNPJ da empresa inválido, entre outros. O Quadro 6 resume a posição dos usuários do modelo proprietário acerca das barreiras e desafios para superação das barreiras ao modelo aberto.

Os benefícios técnicos oferecidos pelo sistema aberto e o poder de algumas das grandes empresas da indústria que o adotaram não foram suficientes para garantir o sucesso do referido modelo na cadeia de suprimentos em análise. Dados de arquivo apontam que a disseminação do modelo aberto atingiu um pequeno percentual (apenas $2 \%$ das empresas da indústria calçadista). A literatura apresenta as barreiras à disseminação da TI no ambiente empresarial que foram identificadas nos casos em estudo. Entre essas, elencam-se: a complexidade e o custo para a implementação de sistemas avançados de TI (FAWCETT et al., 2007); a limitação financeira e/ou restrição de mudança dos processos internos de TI e operacionais (AZEVEDO; CARVALHO, 2012; THIESSE et al., 2011); a cultura corporativa (SILVA; FISCHMANN, 1999), alto custo operacional de setup (JUN; CAI, 2003); a necessidade de um grande número de empresas aderentes ao modelo aberto (AZEVEDO; CARVALHO, 2012). Além desses elementos, o presente estudo identificou o custo operacional advindo do uso de soluções-padrão para codificação e troca de informações e codificação de produtos, como uma variável limitadora para o avanço do sistema aberto de integração da TI, em especial para pequenas empresas; os custos administrativos

Quadro 6. Visão dos usuários do modelo proprietário acerca do modelo aberto.

\begin{tabular}{|l|l|}
\hline \multicolumn{1}{|c|}{ ELEMENTO } & \multicolumn{1}{c|}{ VISÃO } \\
\hline Barreiras ao modelo aberto & $\begin{array}{l}\text { Complexidade de operacionalização; } \\
\text { Falta de equipe técnica para manutenção do modelo. }\end{array}$ \\
\hline Superação das barreiras ao modelo aberto & $\begin{array}{l}\text { Retorno financeiro e/ou operacional; } \\
\text { Plataforma on line para compartilhamento de informações (EDI); } \\
\text { Assessoria para aplicação e manutenção do modelo. }\end{array}$ \\
\hline
\end{tabular}

Fonte: Própria dos autores. 
e de capacitação para o uso dos sistemas abertos influenciam na restrita disseminação do modelo aberto.

Corroborando com a pesquisa, as prestadoras de serviço de TI destacam que a aversão da alta gerência de seus clientes a eventuais mudanças no modelo de gestão das informações se constituiu em outro fator preponderante para o limitado avanço do modelo aberto. A referida aversão advém do fato de que as empresas, em especial as de pequeno porte, utilizam-se dos sistemas de TI apenas para atender às exigências contábeis e fiscais, não visualizando ganhos adicionais com a disseminação da TI nas linhas de produção e processos de expedição/recebimento. Tal problema está alinhado com o trabalho de Jun e Cai (2003) e Murphy e Darley (1999), os quais postulam a resistência à mudança, por parte da alta gerência, como barreira para o avanço de sistemas da TI.

Finalizando, a organização da cadeia de suprimentos calçadista, com a utilização de um modelo aberto de integração da TI, propiciaria ganhos em redes e aumento da eficiência coletiva do arranjo produtivo. Tal afirmação está alinhada com Hayes et al. (2008).

\section{Considerações finais}

O objetivo geral deste artigo foi identificar e compreender os motivos da limitada padronização verificada, a despeito dos benefícios que esta pode trazer para a cadeia de suprimentos calçadista e para a empresa. Foram investigados os constructos integração da TI, padronização dos processos e parceria. O estudo caracterizou-se por ter uma abordagem qualitativa, na qual o método escolhido foi o estudo de caso múltiplo, com propósito exploratório. A operacionalização deu-se por meio de entrevistas (semiestruturadas), análise documental e visita de campo. Os achados advieram de vinte e quatro empresas da cadeia calçadista da região do Vale do Rio dos Sinos, Rio Grande do Sul - Brasil. Foram investigados oito fornecedores de componentes (primeiro nível, ex.: tacos, fivelas e adereços); oito empresas de manufatura de calçado (calçadistas); quatro organizações de varejo; e quatro prestadoras de serviço de TI. A análise dos achados desvelou ganhos, problemas e indutores dos modelos de integração da TI na referida cadeia de suprimentos calçadista.

Relativo, a resposta à questão de pesquisa que norteia o presente estudo, "Como se desenvolve a disputa pelo padrão dominante de gestão da informação na cadeia de suprimentos calçadista?" Desvelou-se que a instigação pelo emprego do modelo proprietário de integração da TI dá-se pela proposta de "oferta" de um modelo simples e barato. Somado a isto, um modelo com reduzido número de informações transitadas entre as empresas e baixo custo de implementação e operacionalização. Esses elementos parecem justificar a grande penetração do referido modelo no setor estudado. Em contrapartida, a incitação pela utilização do modelo aberto dá-se pela proposta de maior organização dos processos internos de gestão da TI, gestão de estoques e produção. Em adição, facilidade no fluxo de informações e materiais na cadeia de suprimentos calçadista. Embora tecnicamente mais avançada, a complexidade de adoção e os custos dessa solução têm limitado a penetração desta na cadeia de suprimentos estudada.

A abrangência geográfica e do perfil das empresas que fazem parte da pesquisa se constituíram em uma limitação para este estudo. As empresas pesquisadas no presente estudo fazem parte da região do Vale do Rio dos Sinos - Rio Grande do Sul - Brasil. Como exemplo desta limitação, cita-se a extensão da pesquisa, na qual foram investigados fornecedores de primeiro nível na cadeia de suprimentos calçadista (fornecedores de taco, adereços, fivelas, adesivos e couro), mas não se estendeu ao próximo nível da referida cadeia de suprimentos.

Em face das oportunidades identificadas diante deste estudo, como proposição para trabalhos futuros, sugere-se investigar a relação entre a integração do fluxo físico, financeiro e da informação e suas complementaridades entre si, e seu impacto no diferencial do desempenho da empresa e da cadeia de suprimentos com a utilização dos modelos de integração da TI estudados no presente trabalho. Futuros esforços podem explorar ainda o impacto da personalização da TI, em um esforço para obter maior compreensão da contribuição aos parceiros na integração interfirmas.

\section{Referências}

AVIV, Y. On the benefits of collaborative forecasting partnerships between retailers and manufacturers. Management Science, p. 777-794, 2007. http://dx.doi. org/10.1287/mnsc.1060.0654

AZEVEDO, S. G.; CARVALHO, H. Contribution of RFID technology to better management of fashion supply chains. International Journal of Retail \& Distribution Management, v. 40, n. 2, p. 128-156, 2012. http:// dx.doi.org/10.1108/09590551211201874

BALLOU, R. H. G. New Managerial Challenges from Supply Chain Opportunities. Industrial Marketing Management, v. 29, p. 7-18, 2000. http://dx.doi. org/10.1016/S0019-8501(99)00107-8

BALOCCO, R. et al. RFID adoption in the FMCG supply chain: an interpretative framework. Supply Chain Management: An International Journal, v. 16, p. 299-315, 2011. http://dx.doi. org/10.1108/13598541111155820

BARDIN, L. Análise de conteúdo. Tradução de Luís Antero Reto e Augusto Pinheiro. Lisboa, 2002.

BARLAS, Y.; GUNDUZ, B. Demand forecasting and sharing strategies to reduce fluctuations and the bullwhip effect in supply chains. Journal of the Operational Research Society, v. 62, p. 458-473, 2011. http://dx.doi. org/10.1057/jors.2010.188 
BEIMBORN, D. et al. The role of process standardization in achieving IT business value. In: HAWAII INTERNATIONAL CONFERENCE ON SYSTEM SCIENCES, 42., 2009, Big Island. Proceedings... Big Island, 2009.

BOECK, H.; WAMBA, S. F. RFID and buyer-seller relationships in the retail supply chain. International Journal of Retail \& Distribution Management, v. 36, n. 6, p. 433-460, 2008. http://dx.doi. org/10.1108/09590550810873929

CACHON, G. P.; FISHER, M. Supply Chain Inventory Management and the Value of Shared Information. Management Science, v. 46, n. 8, p. 1032-1048, 2000. http://dx.doi.org/10.1287/mnsc.46.8.1032.12029

CHEN, M. C.; YANG, T.; LI, H.C. Evaluating the supply chain performance of IT-based inter-enterprise collaboration". Information and Management, v. 44, p. 524-534, 2007. http://dx.doi.org/10.1016/j. im.2007.02.005

CHOPRA, S.; MEINDL, P. Supply Chain Management: Strategy, Planning, and Operation. Prentice-Hall, 2007.

CHUNG, S. H.; TANG, H.; AHMAD, I. Modularity, Integration and IT Personnel Skills Factors in Linking ERP to SCM Systems. Journal of Technology Management \& Innovation, v. 6, 2011.

FAWCETT, S. E. et al. Information Sharing and supply chain performance: the role of connectivity and willingness. Supply Chain Management: An International Journal, v. 12, p. 358-68, 2007. http:// dx.doi.org/10.1108/13598540710776935

FIALA, P. Information sharing in supply chains. Omega, v. 33, p. 419-423, 2005. http://dx.doi.org/10.1016/j. omega.2004.07.006

FLEURY, A. et al. Metodologia de pesquisa em engenharia de produção e gestão de operações. 2 . ed. Rio de Janeiro: Elsevier, 2012.

GODINHO FILHO, M.; FERNANDES, F. C. F.; LIMA, A. D. Pesquisa em Gestão da Produção na indústria de calçados: revisão, classificação e análise. Gestão e Produção, v. 16, n. 2, p. 163-186, 2009. http://dx.doi. org/10.1590/S0104-530X2009000200002

GOL. Resenha sobre o Selo GOL. Disponível em: <http:// www.gol.org.br/>. Acesso em: 20 mar. 2012.

GUNASEKARAN, A.; PATEL, C.; TIRTIROGLU, E. Performance measures and metrics in a supply chain environment. International Journal of Operations and Production Management, v. 21, p. 71-87, 2001. http://dx.doi.org/10.1108/01443570110358468

HALLDORSSON, A. et al. Complementary theories to supply chain management. Supply Chain Management: An International Journal, v. 12, n. 4, p. 284-296, 2007. http://dx.doi.org/10.1108/13598540710759808

HASSINI, E.; SURTI; C.; SEARCY, C.; A literature review and a case study of sustainable supply chains with a focus on metrics. International Journal Production Economics, v.140, p. 69-82, 2012. http://dx.doi. org/10.1016/j.ijpe.2012.01.042

HAYES, R. et al. Produção, estratégia e tecnologia: em busca da vantagem competitiva. Porto Alegre: Bookman, 2008.

HILL, C. A.; SCUDDER, G. D. Supply chain coordination using EDI with performance implications. International Journal of Manufacturing Technology and
Management, v. 19, p. 6-26, 2010. http://dx.doi. org/10.1504/IJMTM.2010.029448

HILSDORF, W. C.; ROTONDARO, R. G.; PIRES, S. R. I. Integração de processos na cadeia de suprimentos e desempenho do serviço ao cliente: um estudo na indústria calçadista de Franca. Gestão \& Produção, v. 16, n. 2, p. 232-244, abr./jun. 2009. http://dx.doi.org/10.1590/ S0104-530X2009000200007

HSIEH, A.; CHOU, C.; CHEN, C. Job standardization and service quality: a closer look at the application of total quality management to the public sector. Total Quality Management, v. 13, n. 7, p. 899-912, 2002. http://dx.doi.org/10.1080/0954412022000017012

JANG, Y.; LEE, J. Factors influencing the success of management consulting projects. International Journal of Project Management, v. 16, n. 2, p. 67-72, 1998. http://dx.doi.org/10.1016/S0263-7863(97)00005-7

JAYARAM, J.; VICKERY, S. K.; DROGE, C. The effects of information systems infrastructure and process improvements on supply-chain time performance. International Journal of Physical Distribution \& Logistics Management, v. 30, n. 3, p. 314-30, 2000. http://dx.doi.org/10.1108/09600030010326082

JEONG, I.; LEON, V. J. A serial supply chain of newsvendor problem with safety stocks under complete and partial information sharing. International Journal of Production Economics, p. 412-419, 2012. http:// dx.doi.org/10.1016/j.ijpe.2011.08.015

JOHANSSON, O.; PALSSON, H. The impact of Auto-ID on logistics performance A benchmarking survey of Swedish manufacturing industries. Benchmarking: An International Journal, v. 16, n. 4, p. 504-522, 2009.

JUN, M.; CAI, S. Key obstacles to EDI success: from the US small manufacturing companies perspective. Industrial Management \& Data Systems, p. 192-203, 2003. http:// dx.doi.org/10.1108/02635570310465670

KÄRKKÄINEN, M.; HOLMSTRÖM, J. Wireless product identification: enabler for hadling efficiency, customization and information sharing. Supply Chain Management: An international journal, v. 7, n. 4, p. 242-252, 2002.

LEE, H. L.; SO, K. C.; TANG, C. S. The Value of Information Sharing in a Two-Level Supply Chain. Management Science, v. 46, n. 5, p. 626-643, 2000. http://dx.doi. org/10.1287/mnsc.46.5.626.12047

LI, S. et al. Development and validation of a measurement instrument for studying supply chain management practices. Journal of Operations Management, v. 23, p. 618-641, 2005. http://dx.doi.org/10.1016/j. jom.2005.01.002

MALHOTRA, A.; GOSAIN,S.; EL SAWY,O. A. Absorptive capacity configurations in supply chains: Gearing for partner-enabled market knowledge creation. MIS Quarterly: Management Information Systems, p. 145-187, 2005.

McLAREN, T.; HEAD, M.; YUAN, Y. Supply chain collaboration alternatives: Understanding the expected costs and benefits. Internet Research, p. 348-364, 2002. http://dx.doi.org/10.1108/10662240210438416

MÜNSTERMANN, B.; ECKHARDT, A.; WEITZEL, $\mathrm{T}$. The performance impact of business process standardization: An empirical evaluation of the recruitment process. Business Process Management 
Journal, v. 16, n. 1, p. 29-56, 2010. http://dx.doi. org/10.1108/14637151011017930

MURPHY, P. R.; DARLEY, J. M. EDI benefits and barriers: comparing international freight forwarders and their customers. International Journal of Physical Distribution \& Logistics Management, v. 29, n. 3, p. 207-16, 1999. http://dx.doi. org/10.1108/09600039910268700

NATIVI, J. J.; LEE, S. Impact of RFID informationsharing strategies on a decentralized supply chain with reverse logistics operations. International Journal of Production Economics, p. 366-377, 2012. http://dx.doi. org/10.1016/j.ijpe.2011.12.024

PALSSON, H.; JOHANSSON, O. The impact of Auto-ID on logistics performance A benchmarking survey of Swedish manufacturing industries. Benchmarking: An International Journal, v. 16, n. 4, p. 504-522, 2009.

PEREIRA. G. M.; SELLITTO, M. A.; BORCHARDT, M. Alterações nos fatores de competição da indústria calçadista exportadora devido à entrada de competidores asiáticos. Produção, v. 20, n. 2, p. 149-159, 2010. http://dx.doi.org/10.1590/S0103-65132010005000022

POWER, D. Strategy development processes as determinants of B2B e-commerce performance: A comparative model in a supply chain management context. Internet Research, p. 557-581, 2005. http://dx.doi. org/10.1108/10662240510629493

RAI, A.; PATNAYAKUNI, R.; SETH, N. Firm performance impacts of digitally enabled supply chain integration capabilities. MIS Quarterly: Management Information Systems, p. 225-246, 2006.

SAHIN, F.; ROBINSON JUNIOR, E. P. Information sharing and coordination in make-to-order supply chains. Journal of Operations Management, v. 23, p. 579- 598, 2005. http://dx.doi.org/10.1016/j.jom.2004.08.007

SANDERS, N. Pattern of information technology use: The impact on buyer-suppler coordination and performance. Journal of Operations Management, p. 349-367, 2008. http://dx.doi.org/10.1016/j.jom.2007.07.003

SANDERS, N. R. Pattern of information technology use: The impact on buyer-suppler coordination and performance. Journal of Operations Management, p. 349-367, 2005.

SEYAL, A. H.; RAHMAN, M. N. A.; MOHAMMAD, H. A. Y. A. A quantitative analysis of factors contributing electronic data interchange adoption among Bruneian SMEs: A pilot study. Business Process Management Journal, v. 13, n. 5, p. 728-746, 2007. http://dx.doi. org/10.1108/14637150710823183

SHAMIR, N. Strategic information sharing between competing retailers in a supply chain with endogenous wholesale price. International Journal of Production Economics, p. 352-365, 2012. http://dx.doi.org/10.1016/j. ijpe.2011.12.023

SILVA, A. L.; FISCHMANN, A. A. Impacto da tecnologia de informação no Supply Chain Management: um estudo multicaso sobre a adoção de EDI entre varejo e indústria agroalimentar. Gestão \& Produção, v. 6, n. 3, p. 201-218, dez. 1999. http://dx.doi.org/10.1590/ S0104-530X1999000300006

SMITH, I. C.; DUCHESSI, P.; GARCIA, J. R. G. Information sharing and business systems leveraging in supply chains: An empirical investigation of one web-based application.
Information \& Management, p. 58-67, 2012. http:// dx.doi.org/10.1016/j.im.2011.12.001

SRINIVASAN, M.; MUKHERJEE, D.; GAUR, A. S. Buyer-supplier partnership quality and supply chain performance: Moderating role of risks, and environmental uncertainty. European Management Journal, v. 29, p. 260-271, 2011. http://dx.doi.org/10.1016/j. emj.2011.02.004

SWAMINATHAN, J. M.; TAYUR, S. R. Models for Supply Chains in E-Business. Management Science, v. 49, n. 10 , p. 1387-1406, 2003. http://dx.doi.org/10.1287/ mnsc.49.10.1387.17309

TANG, O.; MUSA, S. N.; Identifying risk issues and research advancements in supply chain risk management. International Journal Production Economics, v. 133, p. 25-34, 2011. http://dx.doi.org/10.1016/j. ijpe.2010.06.013

THIESSE, F. et al. The rise of the "next-generation bar code": an international RFID adoption study. Supply Chain Management: An International Journal, v. 16, p. 328-345, 2011. http://dx.doi. org/10.1108/13598541111155848

TRKMAN, P. et al. Process approach to supply chain integration. Supply Chain Management: An International Journal, v. 12, n. 2, p. 116-128, 2007. http://dx.doi.org/10.1108/13598540710737307

UNGAN, M. C. Standardization through process documentation. Business Process Management Journal, v. 12 n. 2, p. 135-148, 2006. http://dx.doi. org/10.1108/14637150610657495

WANG, E. T. G.; TAI, J. C. F.; WEI, H.-L. A virtual integration theory of improved supply-chain performance. Journal of Management Information Systems, v. 23, n. 2, p. 41-64. 2006. http://dx.doi.org/10.2753/ MIS0742-1222230203

WU, Y. N.; CHENG, T. C. E. The impact of information sharing in a multiple-echelon supply chain. International Journal of Production Economics, p. 1- 11, 2008. http://dx.doi.org/10.1016/j.ijpe.2008.02.016

YU, Z.; YAN, H.; CHENG, T.C.E. Benefits of information sharing with supply chain partnerships. Industrial Management and Data Systems, v. 101, n. 3, p. 114-121. 2001. http://dx.doi. org/10.1108/02635570110386625

YU, Y.; WANG, Z.; LIANG, L. A vendor managed inventory supply chain with deteriorating raw materials and products. International Journal of Production Economics, p. 266-274, 2007.

YU, Y.; WANG, Z.; LIANG, L. A vendor managed inventory supply chain with deteriorating raw materials and products. International Journal of Production Economics, p. 266-274, 2012. http://dx.doi.org/10.1016/j. ijpe.2011.11.029

YUE, X.; LIU, J. Demand forecast sharing in a dualchannel supply chain. Production, Manufacturing and Logistics, v. 174, p. 646-667, 2006.

ZHOU, H.; BENTON JUNIOR, W. C. Supply chain practice and information sharing. Journal of Operations Management, v. 25, p. 1348-1365, 2007. http://dx.doi. org/10.1016/j.jom.2007.01.009 